\title{
Convergence of iterates of Lasota-Mackey-Tyrcha operators
}

\author{
by Wojciech Bartoszek (Pretoria)
}

\begin{abstract}
We provide sufficient and necessary conditions for asymptotic periodicity of iterates of strong Feller stochastic operators.

1. Let $(X, d)$ be a locally compact, metric, Polish space and $\mathcal{B}$ denote the $\sigma$-algebra of Borel subsets in $X$. Given a $\sigma$-finite measure $\mu$ on $(X, \mathcal{B})$ we denote by $\left(L^{1}(\mu),\|\cdot\|\right)$ the Banach lattice of $\mu$-integrable functions on $X$. Functions which are equal $\mu$-almost everywhere are identified. A linear operator $P$ on $L^{1}(\mu)$ is called stochastic (or Markov according to Lasota's terminology) if

$$
P f \geq 0 \text { and } \int_{X} P f d \mu=1
$$

for all nonnegative and normalized (densities) $f \in L^{1}(\mu)$. The convex set of all densities is denoted by $\mathcal{D}_{\mu}$ (simply $\mathcal{D}$ if $X=\mathbb{R}_{+}$and $\mu$ is the Lebesgue measure on $\mathbb{R}_{+}$). If there exists a Borel measurable function $k: X \times X \rightarrow \mathbb{R}_{+}$ such that

$$
P f(x)=\int_{X} k(x, y) f(y) d \mu(y)
$$

then $P$ is called a kernel operator.

We notice that each kernel stochastic operator may be extended to the Banach lattice $M(X)$ of all bounded signed Borel measures on $(X, \mathcal{B})$. Namely, if $\nu \in M(X)$ and $A$ is Borel we define

1991 Mathematics Subject Classification: Primary 47B38, 47B65, 47G10; Secondary 45D05, 60J05.

Key words and phrases: stochastic (Markov) operator, strong Feller kernel, stationary density, asymptotic periodicity.

I thank the Foundation for Research Development for financial support.
\end{abstract}




$$
P \nu(A)=\int_{X} \int_{X} k(x, y) \mathbf{1}_{A}(x) d \mu(x) d \nu(y) .
$$

Obviously $P \nu \in L^{1}(\mu)$.

The paper is particularly devoted to stochastic kernel operators on $L^{1}\left(\mathbb{R}_{+}\right)$with kernels

$$
k(x, y)= \begin{cases}-\frac{\partial}{\partial x} H(Q(\lambda(x))-Q(y)) & \text { if } 0 \leq y \leq \lambda(x) \\ 0 & \text { otherwise }\end{cases}
$$

They appear in mathematical modelling of the cell cycle. A systematic study of the asymptotic properties of iterates of $(*)$ is being continued by Lasota and his collaborators. The reader is referred to [6] for a comprehensive and updated review of the subject. Here we shall concentrate on the mathematical side rather than on biological applications. Our paper often refers to [1]. Following it we shall assume:

(H) $\quad H:[0, \infty) \rightarrow[0, \infty)$ is nonincreasing and absolutely continuous, $H(0)=1$ and $\lim _{x \rightarrow \infty} H(x)=0$,

$(\mathrm{Q} \lambda) \quad Q:[0, \infty) \rightarrow[0, \infty)$ and $\lambda:[0, \infty) \rightarrow[0, \infty)$ are nondecreasing, absolutely continuous, $Q(0)=\lambda(0)=0$ and $\lim _{x \rightarrow \infty} Q(x)=$ $\lim _{x \rightarrow \infty} \lambda(x)=\infty$.

The class of stochastic operators $P$ with kernels $(*)$ satisfying $(\mathrm{H})$ and $(\mathrm{Q} \lambda)$ is denoted by LMT (Lasota, Mackey, Tyrcha (cf. [7]) who contributed much to the discussed matters). It has recently been proved in [1] that if a LMT stochastic operator $P$ additionally satisfies:

( $\alpha) \quad \int_{0}^{\infty} x^{\alpha} h(x) d x<\liminf _{x \rightarrow \infty} Q(\lambda(x))^{\alpha}-Q(x)^{\alpha}$ for some $0<\alpha \leq 1$, where $h(x)=-d H(x) / d x$ for almost all $x$, and

(c) there exists a nonnegative $c$ such that $h(x)>0$ for almost all $x \geq c$,

then there exists a unique $f_{*} \in \mathcal{D}$ such that

$$
\lim _{n \rightarrow \infty}\left\|P^{n} f-f_{*}\right\|=0 \quad \text { for all } f \in \mathcal{D}
$$

( $P$ is asymptotically stable). In this paper we drop condition (c) and prove that the iterates of a LMT operator with $(\mathrm{H}),(\mathrm{Q} \lambda)$ and $(\alpha)$ are strong operator topology convergent to a finite-dimensional projection (with a slight abuse of the terminology such operators are also called stable (cf. [9])).

We begin with considering a general case. Let us recall (cf. [10]) that a kernel stochastic operator $P$ on $L^{1}(\mu)$ is called strong Feller in the strict sense if 


$$
X \ni y \rightarrow k(\cdot, y) \in \mathcal{D}_{\mu} \quad \text { is } L^{1} \text {-norm continuous. }
$$

Note that (SFS) implies the continuity of $P^{*} h$, where $h \in L^{\infty}(\mu)$ and $P^{*}$ stands for the adjoint operator. This easily follows from $P^{*} h(y)=$ $\int_{X} k(x, y) h(x) d \mu(x)$. It is also well known that if $X$ is compact then (SFS) kernel stochastic operators are compact (see [10]). More details concerning asymptotic properties of iterates of compact (or quasi-compact) positive contractions on Banach lattices can be found in [3] and [4].

If $X$ is not compact then (SFS) does not guarantee automatically any regularity of the trajectories $P^{n} f$. For instance, it may happen that for some $f \in \mathcal{D}_{\mu}$ the sequence $P^{n} f$ converges to a density, while for other $f$ we have $\int_{K} P^{n} f d \mu \rightarrow 0$ for every compact $K \subset X$. Roughly speaking, starting from "good" states the process is rather concentrated, but starting from "bad" states it escapes to "infinity". Also all mixed situations may occur. The so-called Doeblin condition is never satisfied if the transition kernels $k(\cdot, \cdot)$ do not allow "long jumps" (i.e. if $d(y, z) \rightarrow \infty$ implies $\left.\int_{X}|k(x, y)-k(x, z)| d \mu(x) \rightarrow 0\right)$.

For noncompact $X$, in order to obtain asymptotic regularity of iterates of (SFS) stochastic operators, we must impose some extra assumptions. Following [5] we say that a stochastic operator $P$ on $L^{1}(\mu)$ is asymptotically periodic if there exist densities $g_{1}, \ldots, g_{r} \in L^{1}(\mu)$ with disjoint supports, functionals $\Lambda_{1}, \ldots, \Lambda_{r}$ on $L^{1}(\mu)$ and a permutation $\alpha$ of $\{1, \ldots, r\}$ so that for all $f \in L^{1}(\mu)$ we have

$$
\lim _{n \rightarrow \infty}\left\|P^{n} f-\sum_{j=1}^{r} \Lambda_{j}(f) g_{\alpha^{n}(j)}\right\|=0 .
$$

$P$ is said to be constrictive if there exists an $L^{1}$-norm compact set $\mathcal{F} \subseteq \mathcal{D}_{\mu}$ such that $\operatorname{dist}\left(P^{n} f, \mathcal{F}\right) \rightarrow 0$ for all $f \in \mathcal{D}_{\mu}$. It has been proved in [5] that each constrictive stochastic operator $P$ on $L^{1}(\mu)$ is asymptotically periodic.

Given a (SFS) stochastic operator $P$ on $L^{1}(\mu)$ we identify here an invariant sublattice on which $P$ is asymptotically periodic. This sublattice appears to be trivial exactly when for each compact $K \subseteq X$ there exists $f \in \mathcal{D}_{\mu}$ such that

$$
\lim _{N \rightarrow \infty} \frac{1}{N} \sum_{j=1}^{N-1} \int_{K} P^{j} f d \mu=0 .
$$

Condition (SFS) is usually easy to verify. We remark that many important kernels used in mathematical modelling of biological systems have this property. For instance, using [8], Theorem 7.4.8, we easily check that if $y_{n} \rightarrow y$ then 


$$
\begin{aligned}
\int_{0}^{\infty} \mid \frac{\partial}{\partial x} & H(Q(\lambda(x))-Q(y))-\frac{\partial}{\partial x} H\left(Q(\lambda(x))-Q\left(y_{n}\right)\right) \mid d x \\
& =\int_{0}^{\infty}\left|h(Q(\lambda(x))-Q(y))-h\left(Q(\lambda(x))-Q\left(y_{n}\right)\right)\right|(Q \circ \lambda)^{\prime}(x) d x \\
& =\int_{0}^{\infty}\left|h(t-Q(y))-h\left(t-Q\left(y_{n}\right)\right)\right| d t \underset{n \rightarrow \infty}{\longrightarrow} 0,
\end{aligned}
$$

with our convention that $h(x) \equiv 0$ if $x \leq 0$. Hence LMT operators satisfy (SFS).

2. The purpose of this section is to show asymptotic periodicity of (SFS) operators. The reader can view it as a generalization of [2].

We denote by $C_{0}(X)$ the Banach lattice of all continuous functions $h$ on $X$ such that for every $\varepsilon>0$ there exists a compact set $E_{\varepsilon} \subseteq X$ such that $|h(x)| \leq \varepsilon$ for all $x \notin E_{\varepsilon}$ (endowed with the ordinary sup-norm $\|\cdot\|_{\text {sup }}$ ). Given a stochastic operator $P$ we denote by $F$ the minimal (modulo sets of measure zero) measurable set which carries supports of all $P$-invariant densities (its existence follows from separability of the $L^{1}(\mu)$ ). Obviously $L^{1}(F)$ is $P$-invariant.

The next result, which will be the main ingredient of the proofs in Section 3 , is also of some independent interest.

THEOREM 1. Let $P$ be a $(S F S)$ stochastic operator on $L^{1}(\mu)$ such that $P^{*}$ preserves $C_{0}(X)$. If

(i) there exists a compact set $K \subseteq X$ such that

$$
\varlimsup_{N \rightarrow \infty} \frac{1}{N} \int_{K} \sum_{j=0}^{N-1} P^{j} f d \mu>0 \quad \text { for all } f \in \mathcal{D}_{\mu},
$$

then $F$ is nontrivial and $P$ is asymptotically periodic on $L^{1}(F)$. In particular, there are only finitely many P-invariant ergodic densities.

Proof. The set of all subprobabilistic positive measures on $X$ is a compact convex set with respect to the vague topology (we say that a variation norm bounded sequence of measures $\nu_{n}$ is vaguely convergent to $\nu$ if $\lim _{n \rightarrow \infty} \int_{X} h d \nu_{n}=\int_{X} h d \nu$ for all $\left.h \in C_{0}(X)\right)$. Given $f \in \mathcal{D}_{\mu}$ we may choose a sequence $n_{k} \nearrow \infty$ so that the measures with densities

$$
\frac{1}{n_{k}} \sum_{j=0}^{n_{k}-1} P^{j} f=A_{n_{k}} f
$$

are vaguely convergent. By (i) the limit $\nu$ is nonzero and $P A_{n_{j}} f$ tends to 
$P \nu$ vaguely. Since

$$
\left\|A_{n_{k}} f-P A_{n_{k}} f\right\|=\left\|\frac{P^{n_{k}} f-f}{n_{k}}\right\| \underset{k \rightarrow \infty}{\longrightarrow} 0
$$

we conclude that $\nu=P \nu \in L^{1}(\mu)$ is a fixed point of $P$. Normalizing $\nu$ if necessary we obtain a $P$-invariant density.

Now we show that the linear subspace (sublattice) Fix $(P)$ of all $P$ invariant functions is finite-dimensional. Assume we are given pairwise orthogonal $P$-invariant densities $f_{1}, \ldots, f_{k}$. By (i) we have $\int_{K} f_{j} d \mu>0$. Consider the following family of (restricted to $K$ ) continuous functions:

$$
g_{j}=\left.\left(P^{*} \mathbf{1}_{F_{j}}\right)\right|_{K}, \quad \text { where } \quad F_{j}=\operatorname{supp}\left(f_{j}\right) .
$$

Clearly

$$
g_{j}(x)=1 \quad \text { for all } x \in \bar{F}_{j} \cap K,
$$

and

$$
g_{j}(x)=0 \quad \text { if } x \in \bigcup_{l \neq j} \bar{F}_{l} \cap K .
$$

As a result, $\left\|g_{j}-g_{l}\right\|_{\text {sup }}=1$ for $j \neq l$. The condition (SFS) combined with the Arzelà theorem easily gives $\|\cdot\|_{\text {sup }}$-compactness of $\left.\overline{P^{*} B_{1}}\right|_{K}$, where $B_{1}$ stands for the unit ball of $L^{\infty}(\mu)$. Hence, $k$ is bounded and there are only finitely many ergodic $P$-invariant densities $f_{1}, \ldots, f_{r}$.

For fixed $1 \leq j \leq r$ we show that $P$ is asymptotically periodic on $L^{1}\left(F_{j}\right)$. First we notice that each trajectory

$$
\gamma(f)=\left\{P^{n} f\right\}_{n \geq 0}, \quad \text { where } f \in L^{1}\left(F_{j}\right),
$$

is $L^{1}$-norm relatively compact. We may confine discussion to $0 \leq f \leq f_{j}$. Clearly $\gamma(f)$ is weakly compact, which follows from invariance and weak compactness of the order interval $\left[0, f_{j}\right]=\left\{f \in L^{1}\left(F_{j}\right): 0 \leq f \leq f_{j}\right\}$ (see [11], II.5.10). Let $P^{n_{l}} f$ be an arbitrary sequence. We choose a subsequence $P^{n_{l_{m}}} f$ which is weakly convergent to $\tilde{f}$. Suppose $P^{n_{l_{m}}} f$ is not norm relatively compact. Choosing a further subsequence if necessary we may assume that

$$
\left\|P^{n_{l_{m+1}}+1} f-P^{n_{l_{m}}+1} f\right\|>\varepsilon
$$

for some $\varepsilon>0$ and all $m$. By Prokhorov's theorem the sequence of densities $P^{n} f$ is tight. Hence there exists a compact set $K_{\varepsilon} \subseteq X$ such that for all $n$,

$$
\int_{X \backslash K_{\varepsilon}} P^{n} f d \mu \leq \varepsilon / 4 .
$$

Now we find $h_{m} \in L^{\infty}\left(F_{j}\right)$ with $\left|h_{m}\right| \leq 1$ so that

$$
\int_{X} P\left(P^{n_{l_{m+1}}} f-P^{n_{l_{m}}} f\right) h_{m} d \mu>\varepsilon .
$$


Then

$$
\int_{K_{\varepsilon}}\left(P^{n_{l_{m+1}}} f-P^{n_{l_{m}}} f\right) P^{*} h_{m} d \mu \geq \varepsilon / 2 .
$$

As before $\left\{\left.P^{*} h_{m}\right|_{K_{\varepsilon}}\right\}_{m=1}^{\infty}$ is relatively compact for the uniform convergence on $K_{\varepsilon}$. Choosing again a subsequence we may assume that $P^{*} h_{m} \rightarrow h$ uniformly on $K_{\varepsilon}$. This leads to a contradiction as

$$
\begin{aligned}
\varepsilon / 2 & \leq \varlimsup_{m \rightarrow \infty} \int_{K_{\varepsilon}}\left(P^{n_{l_{m+1}}} f-P^{n_{l_{m}}} f\right) P^{*} h_{m} d \mu \\
& =\lim _{m \rightarrow \infty} \int_{K_{\varepsilon}}\left(P^{n_{l_{m}+1}} f-P^{n_{l_{m}}} f\right) h d \mu=0 .
\end{aligned}
$$

We denote by $\Omega_{j}$ the subspace of all $L^{1}$-norm recurrent $f \in L^{1}\left(F_{j}\right)$. It is well known that $\Omega_{j}$ consists of all limit vectors in $L^{1}\left(F_{j}\right)$ (see [3], [4] for all details). Given a sequence $\underline{n}=n_{k} \nearrow \infty$ we denote the by $\Omega_{\underline{n}}$ the closed sublattice of $\Omega_{j}$ consisting of all vectors $f$ which are recurrent along the sequence $n_{k}$ (i.e. $\left\|P^{n_{k}} f-f\right\| \rightarrow 0$ as $k \rightarrow \infty$ ). We notice that regardless of the dimension of $\Omega_{\underline{n}}$, for every compact $C \subseteq X$ the restricted sublattice $\left.\Omega_{\underline{n}}\right|_{C}$ is finite-dimensional. In fact, $\left.\operatorname{dim} \Omega_{\underline{n}}\right|_{C} \leq r_{C}$, where $r_{C}$ denotes the largest $j$ such that there are $0 \leq h_{1}, \ldots, h_{j} \leq 1, h_{l} \in P^{*} B_{1}$, with

$$
\sup _{x \in C}\left|h_{l}(x)-h_{\tilde{l}}(x)\right|=1
$$

for distinct $l, \tilde{l}$ (it follows from (SFS) that $r_{C}$ is finite). Let $\widetilde{g}_{1}=\left.\beta_{1} g_{1}\right|_{C}, \ldots$, $\widetilde{g}_{r_{C}}=\left.\beta_{r_{C}} g_{r_{C}}\right|_{C}$ form a normalized, positive and orthogonal basis in $\left.\Omega_{\underline{n}}\right|_{C}$ (for some $\beta_{l} \geq 1$ and $g_{l} \in \Omega_{\underline{n}}$ ). Given $\varepsilon>0$ we find a compact set $C=\underline{C_{\varepsilon}} \subseteq$ $X$ such that

$$
\int_{C} f_{j} d \mu>1-\varepsilon
$$

It follows from the ergodicity of $f_{j}$ that for each density $g \in \Omega_{\underline{n}}$ we have $A_{n} g \rightarrow f_{j}$ in $L^{1}\left(F_{j}\right)$. Hence there exists $n$ such that

$$
\int_{C} P^{n} g d x>1-\varepsilon
$$

We have

$$
\left.P^{n} g\right|_{C}=\sum_{l=1}^{r_{C}} \alpha_{l} \widetilde{g}_{l}, \quad \text { where } \quad \alpha_{l} \geq 0, \text { and } 1 \geq \sum_{l=1}^{r_{C}} \alpha_{l}>1-\varepsilon .
$$

Equivalently, for each $g \in \Omega_{\underline{n}}$ there is a natural $n$ so that

$$
\operatorname{dist}\left(P^{n} g, \operatorname{conv}\left\{\widetilde{g}_{1}, \ldots, \widetilde{g}_{r_{C}}, 0\right\}\right)<\varepsilon .
$$


Therefore,

$$
\operatorname{dist}\left(P^{n+k} g, \mathcal{F}_{\varepsilon, \underline{n}}\right) \leq \varepsilon \text { for all } k \geq 0,
$$

where $\mathcal{F}_{\varepsilon, \underline{n}}$ denotes the $L^{1}$-norm closure of the set

$$
\left\{\sum_{l=1}^{r_{C}} \alpha_{l} P^{k} \widetilde{g}_{l}: k=0,1,2, \ldots, \sum_{l=1}^{r_{C}} \alpha_{l} \leq 1, \alpha_{l} \geq 0\right\} .
$$

As all trajectories in $L^{1}\left(F_{j}\right)$ are norm relatively compact the set $\mathcal{F}_{\varepsilon, \underline{n}}$ is compact. Clearly it is $P$-invariant. Hence by recurrence of $P^{n} g$ we obtain

$$
\operatorname{dist}\left(g, \mathcal{F}_{\varepsilon, \underline{n}}\right) \leq \varepsilon \text {. }
$$

Since $\varepsilon>0$ is arbitrary, this implies that the set of all densities from $\Omega_{n}$ is relatively compact, and $\Omega_{\underline{n}}$ is finite-dimensional with $\operatorname{dim} \Omega_{\underline{n}} \leq r_{C}$. Moreover, $P$ has a positive inverse on $\Omega_{\underline{n}}$, so from the general theory of Markov operators $P$ permutes vectors of a unique, positive, normalized and orthogonal basis in $\Omega_{\underline{n}}$. In particular, $P$ is periodic (i.e. $P^{\mathrm{d}}=\mathrm{Id}$, where $\mathrm{d}=\mathrm{d}(\underline{n})$ depends on $\underline{n}$ ) on $\Omega_{\underline{n}}$.

For arbitrary $\Omega_{\underline{n}}, \Omega_{\underline{m}}$ we may find $\mathrm{d}$ (for instance $\mathrm{d}=\mathrm{d}(\underline{n}) \cdot \mathrm{d}(\underline{m})$ ) such that $\Omega_{\underline{n}}, \Omega_{\underline{m}} \subseteq \Omega_{\{k \mathrm{~d}\}}$. Hence,

$$
\left.\operatorname{dim} \Omega_{j}\right|_{C}=\operatorname{dim}\left\{\left.f\right|_{C}: f \in \Omega_{j}\right\} \leq r_{C} .
$$

Repeating the arguments applied to $\left.\Omega_{\underline{n}}\right|_{C}$, we construct a compact set $\mathcal{F}_{\varepsilon}$ such that

$$
\operatorname{dist}\left(g, \mathcal{F}_{\varepsilon}\right) \leq \varepsilon \quad \text { for all densities } g \in \Omega_{j} .
$$

Since $\varepsilon$ may be taken as small as we wish, $\Omega_{j}$ is finite-dimensional. For each density $f \in L^{1}\left(F_{j}\right)$ the iterates $P^{n} f$ are attracted to the set $\mathcal{D}_{\mu} \cap \Omega_{j}$, which obviously is norm compact. In particular, $P$ is constrictive. By [5] (see also [2]-[4]), $P$ is asymptotically periodic on $L^{1}\left(F_{j}\right)$. We easily extend this property to $L^{1}(F)$ where $F=\bigcup_{j=1}^{r} F_{j}$.

We want to emphasize that if $P$ satisfies (SFS) and $P^{*}$ preserves $C_{0}(X)$, and $F$ is nontrivial, then for each $f \in L^{1}(F)$ and $\varepsilon>0$ there exists $f_{\varepsilon}$ such that $\left\|f-f_{\varepsilon}\right\| \leq \varepsilon$ and the trajectory $\gamma\left(f_{\varepsilon}\right)$ asymptotically becomes periodic (i.e. $\omega\left(f_{\varepsilon}\right)=\left\{\widetilde{f}: \underline{\lim }_{n \rightarrow \infty}\left\|P^{n} f_{\varepsilon}-\widetilde{f}\right\|=0\right\}$ is finite, and $P$ permutes $\left.\omega\left(f_{\varepsilon}\right)\right)$. Then we may say that $P$ is almost asymptotically periodic on $L^{1}(F)$. In contrast to this, one can show that the substochastic operator $\widetilde{P}$ defined on $L^{1}\left(F^{\mathrm{c}}\right)$ by $\widetilde{P} f=\left.(P f)\right|_{F^{\mathrm{c}}}$ (where $\left.F^{c}=X \backslash F\right)$ is Cesàro sweeping (consult [6] for the terminology). For general $f \in \mathcal{D}_{\mu}$ the asymptotic properties of the trajectory $\gamma(f)$ depend on

$$
\delta(f)=\lim _{n \rightarrow \infty} \int_{F} P^{n} f d \mu .
$$


If $\delta(f)>0$ then an asymptotically nontrivial portion of $P^{n} f$ behaves periodically. The case when the quantity $\delta(f)$ is uniformly separated from 0 , for all $f \in \mathcal{D}$, is discussed below.

COROLlaRY 1. Let $P$ be a kernel stochastic operator on $L^{1}(\mu)$ satisfying $(S F S)$ and such that $P^{*}$ preserves $C_{0}(X)$. Then the following conditions are equivalent:

(i) $P$ is asymptotically periodic on $L^{1}(\mu)$,

(ii) there exist a compact set $K \subseteq X$ and $\delta>0$ such that

$$
\varlimsup_{n \rightarrow \infty} \int_{K} \frac{f+P f+\ldots+P^{n-1} f}{n} d \mu>\delta \quad \text { for all } f \in \mathcal{D}_{\mu} .
$$

Proof. Only (ii) implies (i) needs to be proved. By Theorem 1 it is enough to show that for each $f \in \mathcal{D}_{\mu}$ we have

$$
\lim _{n \rightarrow \infty} \int_{F} P^{n} f d \mu=1
$$

(here we may repeat essentially the same arguments as in the proof of Theorem 1.3 in [1], but for the sake of completeness we provide a full proof). Choosing a subsequence if necessary we may assume that

$$
\left.\left.\left(\frac{1}{n_{k}} \sum_{j=0}^{n_{k}-1} P^{j} f\right)\right|_{K} \underset{k \rightarrow \infty}{\longrightarrow} f_{*}\right|_{K}
$$

in the $L^{1}$-norm, where $f_{*}$ is $P$-invariant. By (ii) we easily get $\delta<\left\|\left.f_{*}\right|_{K}\right\|$. As a result, for every $f \in \mathcal{D}_{\mu}$ there is a natural $n$ so that

$$
\int_{F} P^{n} f d \mu>\delta
$$

Suppose that there exists $f \in \mathcal{D}_{\mu}$ with $\delta(f)<1$. If $m$ is large enough then

$$
\int_{F} P^{m} f d \mu>\delta(f)-\frac{(1-\delta(f)) \delta}{2} \text {. }
$$

Consider

There is $n$ such that

$$
f_{1}=\frac{\mathbf{1}_{F^{\mathrm{c}}} P^{m} f}{\int_{F^{\mathrm{c}}} P^{m} f d \mu}
$$

$$
\int_{F} P^{n} f_{1} d \mu=\frac{1}{\int_{F^{\mathrm{c}}} P^{m} f d \mu} \int_{F} P^{n}\left(\mathbf{1}_{F^{\mathrm{c}}} P^{m} f\right) d \mu>\delta .
$$

Thus, 


$$
\begin{aligned}
\int_{F} P^{n+m} f d \mu & =\int_{F} P^{n}\left(\mathbf{1}_{F} P^{m} f+\mathbf{1}_{F^{\mathrm{c}}} P^{m} f\right) d \mu \\
& =\int_{F} P^{n}\left(\mathbf{1}_{F} P^{m} f\right) d \mu+\int_{F} P^{n}\left(\mathbf{1}_{F^{\mathrm{c}}} P^{m} f\right) d \mu \\
& >\int_{F} P^{m} f d \mu+\delta \int_{F^{\mathrm{c}}} P^{m} f d \mu \\
& \geq \delta(f)-\frac{(1-\delta(f)) \delta}{2}+(1-\delta(f)) \delta \\
& =\delta(f)+\frac{(1-\delta(f)) \delta}{2}>\delta(f),
\end{aligned}
$$

contradicting the definition of $\delta(f)$.

Comment. We remark that all the above results remain valid for $P$ being strongly Feller (i.e. $P^{*} h$ is continuous for all bounded measurable $h$ ). In fact, it is well known (see Theorem 5.9 on p. 37 of [10]) that strong Feller implies (SFS) for $P^{2}$.

3. In this section we study asymptotic properties of the iterates of LMT operators. It has been just noticed that they are strong Feller in the strict sense. Since

if

$$
k(x, y)=-\frac{\partial}{\partial x} H(Q(\lambda(x))-Q(y))=0
$$

$$
x \leq \lambda_{*}^{-1}(y)=\inf \{0 \leq z: \lambda(z)=y\},
$$

and $\lambda_{*}^{-1}(y)$ tends to $\infty$ with $y$, it follows that $P^{*}$ preserves $C_{0}\left(\mathbb{R}_{+}\right)$. Therefore the results of Section 2 are applicable.

TheOREM 2. Let $P$ be a LMT stochastic operator associated with $H, Q, \lambda$. Assume that there exist $a>0$ and $\delta>0$ so that

$$
\varlimsup_{n \rightarrow \infty} \int_{0}^{a} \frac{f+P f+\ldots+P^{n-1} f}{n} d x>\delta \quad \text { for all } f \in \mathcal{D} .
$$

Then

(a) $a_{*}=\sup \{x \geq 0: \lambda(x) \leq x\}<a$,

(b) $\operatorname{Fix}(P)$ is finite-dimensional and $\lim _{n \rightarrow \infty}\left\|P^{n} f-S f\right\|=0$ for all $f \in L^{1}\left(\mathbb{R}_{+}\right)$, where $S$ is a stochastic projection onto $\operatorname{Fix}(P)$,

(c) $\operatorname{dim} \operatorname{Fix}(P) \leq\lceil a / T(P, a)\rceil$, where $T(P, r)=\sup \{t>0$ : if $0 \leq y$, $\widetilde{y} \leq r$ and $|y-\widetilde{y}| \leq t$ then $\|k(\cdot, y)-k(\cdot, \widetilde{y})\|<2\}$ and $\lceil z\rceil$ stands for the smallest natural number greater than or equal to $z$. In particular, $P$ is asymptotically stable if $T(P, a) \geq a$. 
Proof. By Corollary 1 the operator $P$ is asymptotically periodic. If $\lambda(x) \leq x$ then the space $L^{1}([x, \infty))$ is $P$-invariant. By easy calculations,

$$
\begin{aligned}
& P^{*} \mathbf{1}_{[c, d)}(y) \\
& \quad= \begin{cases}H(Q(\lambda(c))-Q(y))-H(Q(\lambda(d))-Q(y)) & \text { if } 0 \leq y<\lambda(c), \\
1-H(Q(\lambda(d))-Q(y)) & \text { if } \lambda(c) \leq y<\lambda(d), \\
0 & \text { if } \lambda(d) \leq y\end{cases}
\end{aligned}
$$

If $\lambda(c) \leq c$ then substituting $d=\infty$ we get

$$
P^{*} \mathbf{1}_{[c, \infty)}(y) \geq \mathbf{1}_{[c, \infty)}(y) \quad \text { for all } y .
$$

Hence the set $\{x: \lambda(x) \leq x\}$ must be bounded and $a_{*}$ is finite. Now it is clear that

$$
\lambda\left(a_{*}\right)=a_{*} \text { and } a_{*}<a .
$$

Let $g_{1}, \ldots, g_{r}$ be a basis of positive, normalized and pairwise orthogonal functions in the space $\Omega$ of all recurrent elements and $g_{1}, \ldots, g_{l}$ be a cycle (i.e. $P g_{j}=g_{j+1}$ for $1 \leq j \leq l$, where $j+1$ is understood modulo $l$ ). Define $D_{j}=\operatorname{supp} g_{j}$ and $c_{j}=\operatorname{essinf} D_{j}$. Then we have

$$
P^{*} \mathbf{1}_{D_{j}}(y)= \begin{cases}1 & \text { if } y \in \bar{D}_{j-1}, \\ 0 & \text { for all } y \in \bar{D}_{s} \text { if } s \neq j-1 .\end{cases}
$$

We may assume that $\max \left\{c_{1}, \ldots, c_{l}\right\}=c_{l}$. Thus,

$$
P^{*} \mathbf{1}_{\left[c_{l}, \infty\right)} \geq P^{*} \mathbf{1}_{D_{l}} \geq \mathbf{1}_{D_{l-1}} .
$$

By continuity,

$$
P^{*} \mathbf{1}_{\left[c_{l}, \infty\right)}\left(c_{l-1}\right)=P^{*} \mathbf{1}_{D_{l}}\left(c_{l-1}\right)=1
$$

Since

$$
P^{*} \mathbf{1}_{\left[c_{l}, \infty\right)}(y)= \begin{cases}H\left(Q\left(\lambda\left(c_{l}\right)\right)-Q(y)\right), & 0 \leq y \leq \lambda\left(c_{l}\right), \\ 1 & \text { otherwise }\end{cases}
$$

we conclude that

$$
H\left(Q\left(\lambda\left(c_{l}\right)\right)-Q(y)\right)=1 \quad \text { for all } c_{l-1} \leq y \leq \lambda\left(c_{l}\right) .
$$

Therefore

$$
P^{*} \mathbf{1}_{\left[c_{l}, \infty\right)} \geq \mathbf{1}_{\left[c_{l-1}, \infty\right)} \geq \mathbf{1}_{\left[c_{l}, \infty\right)} .
$$

This implies that $L^{1}\left(\left[c_{l}, \infty\right)\right)$ is $P$-invariant. Since $g_{1}, \ldots, g_{l}$ form a cycle it is possible only if $c_{1}=c_{2}=\ldots=c_{l}$. Hence $l=1$, since by $(\beta)$ the continuous functions $P^{*} \mathbf{1}_{D_{j}}$ would take values 0 and 1 arbitrary close to $c_{l}$. Repeating the previous discussion for other cycles, one shows that each of them reduces to a singleton, and the convergence

$$
\lim _{n \rightarrow \infty}\left\|P^{n} f-S f\right\|=0
$$


follows. Clearly $S$ is a finite-dimensional stochastic projection onto $\Omega=$ Fix $(P)$. Let $F_{1}, \ldots, F_{r}$ be the supports of ergodic densities. We have

$$
\|k(\cdot, y)-k(\cdot, \widetilde{y})\|=2
$$

if $y, \widetilde{y}$ are taken from distinct sets $F_{j} \cap[0, a]$. This yields the estimate

$$
\operatorname{dim}(S) \leq\lceil 1 / T(P, a)\rceil
$$

Combining [1], Theorem 2.1, with our Theorem 2 we immediately get

Corollary 2. Let $P$ be a LMT stochastic operator and suppose there exist positive $\varepsilon, \varrho$, a and $0<\alpha \leq 1$ such that

$$
\varepsilon+\int_{0}^{\infty} x^{\alpha} h(x) d x<\varrho<Q(\lambda(t))^{\alpha}-Q(t)^{\alpha} \quad \text { for all } t \geq a .
$$

Then there exists a finite-dimensional stochastic projection $S$ such that

$$
\lim _{n \rightarrow \infty}\left\|P^{n} f-S f\right\|=0 \quad \text { for all } f \in L^{1}\left(\mathbb{R}_{+}\right) .
$$

Moreover, $\operatorname{dim}(S) \leq\lceil a / T(P, a)\rceil$.

Proof. By [1] (see the proof of Theorem 2.1) for every $f \in \mathcal{D}$ there exists a natural $n_{0}(f)$ such that

$$
\frac{1}{n} \sum_{j=0}^{n-1} \int_{0}^{a} P^{j} f d x \geq \frac{\varepsilon}{2 M} \quad \text { for all } n \geq n_{0}(f),
$$

where $M=\sup \left\{\left|Q(\lambda(x))^{\alpha}-Q(x)^{\alpha}-\varrho\right|: 0 \leq x \leq a\right\}$. Now we can apply Theorem 2.

\section{References}

[1] K. Baron and A. Lasota, Asymptotic properties of Markov operators defined by Volterra type integrals, Ann. Polon. Math. 57 (1993), 161-175.

[2] W. Bartoszek, Asymptotic stability of the iterates of contractions on Banach lattices, in: Proc. Internat. Conf. on Function Spaces, Poznań 1986, J. Musielak (ed.), Teubner, Leipzig, 1987, 153-157.

[3] - Asymptotic periodicity of the iterates of positive contractions on Banach lattices, Studia Math. 91 (1988), 179-188.

[4] - Asymptotic properties of the iterates of stochastic operators on $(A L)$ Banach lattices, Ann. Polon. Math. 52 (1990), 165-173.

[5] A. Lasota, T. Y. Li and J. A. Yorke, Asymptotic periodicity of the iterates of Markov operators, Trans. Amer. Math. Soc. 286 (1984), 751-764.

[6] A. Lasota and M. C. Mackey, Chaos, Fractals and Noise: Stochastic Aspects of Dynamics, Appl. Math. Sci. 97, Springer, New York, 1993.

[7] A. Lasota, M. C. Mackey and J. Tyrcha, The statistical dynamics of recurrent biological events, J. Math. Biology 30 (1992), 775-800. 
[8] S. Łojasiewicz, An Introduction to the Theory of Real Functions, Wiley, Chichester, 1988.

[9] F. Räbiger, Attractors and asymptotic periodicity of positive operators on Banach lattices, Tübingen Ber. Funktionanal. 3 (1993/94), 184-203.

[10] D. Revuz, Markov Chains, Elsevier, Amsterdam, 1984.

[11] H. H. Schaefer, Banach Lattices and Positive Operators, Springer, New York, 1974 .

Department of Mathematics

University of South Africa

P.O. Box 392

Pretoria 0001, South Africa

E-mail: bartowk@risc5.unisa.ac.za

Reçu par la Rédaction le 20.11.1994

Révisé le 26.4.1995 